\section{Diagnosing the cause of bilateral paediatric cataracts: comparison of standard testing with a next-generation sequencing approach}

\author{
Abstract \\ Purpose In addition to environmental causes \\ such as TORCH infection, trauma and drug \\ or chemical exposure, childhood cataracts \\ (CC) frequently have a genetic basis. They \\ may be isolated or syndromic and have been \\ associated with mutations in over $\mathbf{1 1 0}$ genes. \\ We have recently demonstrated that next- \\ generation sequencing (NGS), a high \\ throughput sequencing technique that \\ enables the parallel sequencing of multiple \\ genes, is ideally suited to the investigation \\ of bilateral CC. This study assesses the \\ diagnostic outcomes of traditional routine \\ investigations and compares this with \\ outcomes of NGS testing. \\ Methods A retrospective review of the \\ medical records of 27 consecutive patients \\ with bilateral CC presenting in 2010-2012 \\ was undertaken. The outcomes of routine \\ investigations in these patients, including \\ TORCH screen, urinalysis, karyotyping, and \\ urinary and plasma organic amino acids, \\ were collated. The success of routine genetic \\ investigations undertaken over 10 years \\ (2000-2010) was also assessed. \\ Results By April 2014, the underlying cause \\ of bilateral CC had been identified in just one \\ of 27 patients despite $44 \%(n=12)$ receiving \\ a full 'standard' investigative work-up and \\ $22 \%(n=6)$ investigations in addition to the \\ standard work-up. Fifteen of these patients \\ underwent NGS testing and nine $(60 \%)$ of \\ these received a diagnosis for their CC. \\ Conclusion The frequency of patients \\ receiving a diagnosis for their $\mathrm{CC}$ after \\ standard care and the time taken to diagnosis \\ was disappointing. NGS testing improved \\ diagnostic rates and time to diagnosis, as well \\ as changing clinical management. These data
}

M Musleh¹, G Hall², IC Lloyd ${ }^{3,4}$, RL Gillespie, S Waller ${ }^{2}$, S Douzgou ${ }^{2}$, J Clayton-Smith ${ }^{2}$, E Kehdi ${ }^{3,4}$, GCM Black ${ }^{1,2}$ and J Ashworth ${ }^{3,4}$ serve as a baseline for future evaluation of novel diagnostic modalities.

Eye (2016) 30, 1175-1181; doi:10.1038/eye.2016.105; published online 17 June 2016

\section{Introduction}

Congenital and developmental cataracts (childhood cataract (CC)) are a major cause of childhood visual deficit, especially in developing countries. In the UK, the incidence of CC presenting in the first year of life has been estimated as 2.49 per 10000 , rising to 3.46 per 10000 by the age of 15 years. ${ }^{1}$ A wide range of systemic conditions are associated with bilateral CCs, including antenatal infection, metabolic disease, chromosomal and single-gene abnormalities. ${ }^{2}$ As a consequence of this, a range of investigations are often performed with the aim of identifying an underlying cause. These include TORCH screen, karyotyping, urinalysis for reducing agents and organic amino acids, measurement of plasma galactokinase levels and basic blood tests (such as full-blood count and liver function tests). ${ }^{3}$ Despite the publication of several care pathways guiding management and investigation of $\mathrm{CC}_{1}^{2,3}$ the proportion of cases of bilateral $\mathrm{CC}$ in which the underlying cause was identified has been shown in the past to be low. Multiple professionals are often involved, with many appointments and investigations needed as part of the traditional care pathway of the child with cataract.

We have recently demonstrated that nextgeneration sequencing (NGS) is an effective methodology for the diagnosis of single-gene disorders causing bilateral CC, identifying the cause in $70-80 \%{ }^{4}$ This indicates its potential to have a significant diagnostic impact for patients.

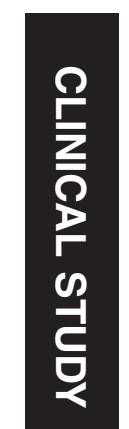

${ }^{1}$ Manchester Centre for Genomic Medicine, Faculty of Medical and Human Sciences, Institute of Human Development, University of Manchester, Manchester Academic Health Science Centre (MAHSC), Saint Mary's Hospital, Manchester, UK

${ }^{2}$ Manchester Centre for Genomic Medicine, Central Manchester University Hospitals NHS Foundation Trust, MAHSC, Saint Mary's Hospital, Manchester, UK

${ }^{3}$ Department of Ophthalmology, Manchester Royal Eye Hospital, Central Manchester Foundation Trust and Manchester Academic Health Science Centre, The University of Manchester, Manchester, UK

${ }^{4}$ Centre for Ophthalmology and Vision Sciences, Faculty of Medical and Human Sciences, Institute of Human Development, University of Manchester, Manchester, UK

Correspondence: GCM Black, Manchester Centre for Genomic Medicine, Faculty of Medical and Human Sciences, Institute of Human Development, University of Manchester, MAHSC, Manchester M13 9WL, UK

Tel: +0161 276 1234; Fax: +0161276 6145 . E-mail: Graeme.Black@ manchester.ac.uk

Received: 20 October 2015 Accepted in revised form: 13 April 2016 Published online: 17 June 2016 
Table 1 Clinical features of patients in baseline cohort

\begin{tabular}{|c|c|c|c|c|}
\hline $\begin{array}{l}\text { Case } \\
\text { number }\end{array}$ & Gender & $\begin{array}{c}\text { Family history of } \\
\text { paediatric cataract/ } \\
\text { parental consanguinity }\end{array}$ & Systemic or isolated paediatric cataract & Cataract phenotype \\
\hline 1 & $\mathrm{~F}$ & No/yes & Isolated & Lamellar \& nuclear \\
\hline 2 & M & No/no & Isolated & Nuclear \\
\hline 3 & $\mathrm{~F}$ & Yes/no & Isolated & Sutural \& lamellar \\
\hline 4 & $\mathrm{~F}$ & Yes/no & Isolated & Dense nuclear \\
\hline 5 & M & Yes/no & Isolated & Lamellar \\
\hline 6 & $\mathrm{M}$ & No/no & Isolated & Cortical \& pulverulent \\
\hline 7 & $\mathrm{~F}$ & Yes/no & Isolated & Lamellar \\
\hline 8 & M & Yes/no & Isolated & Lamellar \\
\hline 9 & $\mathrm{~F}$ & No/no & Systemic (learning disability, LD) & Lamellar \\
\hline 10 & $\mathrm{M}$ & No/yes & Systemic (LD) & Total cataract \\
\hline 11 & $\mathrm{~F}$ & Yes/no & Isolated & Anterior sutural \\
\hline 14 & $\mathrm{~F}$ & No/no & Isolated & Nuclear \\
\hline 15 & $\mathrm{~F}$ & $\mathrm{No} / \mathrm{no}$ & Systemic (LD and cerebellar hypoplasia) & Sutural \& nuclear \\
\hline 16 & $\mathrm{M}$ & No/yes & Isolated & Nuclear \\
\hline 17 & $\mathrm{~F}$ & No/no & Systemic (epilepsy, LD and recurrent fractures) & Posterior \& cortical \\
\hline 18 & $\mathrm{~F}$ & No/yes & Isolated & Posterior \\
\hline 19 & M & No/no & Isolated & Lamellar \\
\hline 20 & M & No/no & Systemic (LD and microcephaly) & Posterior capsular \\
\hline 21 & $\mathrm{M}$ & Yes/no & Isolated & Nuclear, cortical \& posterior lenticonus \\
\hline 22 & $\mathrm{M}$ & No/yes & Systemic (cardiac abn) & Total cataract \\
\hline 23 & M & No/no & Systemic (LD, dysmorphic and hypospadias) & Lamellar \& nuclear \\
\hline 24 & M & No/no & Systemic (LD, pulmonary stenosis and hypothyroidism) & Lamellar \\
\hline 25 & M & Yes/no & Isolated & Stellate central cataract \\
\hline 26 & M & No/no & Systemic (LD and cerebellar hypoplasia) & Total cataract \\
\hline 27 & M & $\mathrm{No} / \mathrm{no}$ & Systemic (LD, cirrhosis, and spastic diplegia) & Lamellar \\
\hline
\end{tabular}

To evaluate the efficacy of the current standard care pathways, the effectiveness of the clinical investigations routinely undertaken to identify an underlying cause of bilateral CC were investigated. Such data are valuable as a baseline against which to assess future clinical and economic impacts of incorporating NGS into clinical diagnostic pathways.

\section{Materials and methods}

\section{Measurement of baseline care}

A retrospective case note review was undertaken of 27 consecutive paediatric patients first diagnosed with bilateral CC at age 12 years and under. These patients were identified through the paediatric ophthalmic surgical list of a single consultant at the Manchester Royal Eye Hospital (MREH) from November 2010-2012 (Tables 1 and 2). Cases of unilateral cataract were excluded from this study. Outcomes of clinical investigations were assessed up until 1 April 2014. This research adhered to the tenets of the Declaration of Helsinki.

Patients were categorized into groups based on investigations undertaken. The 'standard care' group underwent recommended investigation used to determine the cause of CC as outlined in existing care pathways. These investigations were TORCH screen (including plasma Ig or PCR investigations), karyotype, urinalysis for reducing substances and organic amino acids. The 'extensive investigations group' received additional investigations in addition to the basic standard work-up. This list included array comparative genomic hybridization (aCGH, microarray), single-gene sequencing, fluorescent in situ hybridization and biochemical studies (Table 2).

A further retrospective review was undertaken of the outcomes of all cytogenetic (karyotype/microarray) investigations conducted on congenital and developmental cataract patients treated at the MREH between 2000 and 2010. Results were divided into those with negative (normal) findings and those reporting positive (abnormal) findings (Figure 1).

A retrospective analysis of TORCH screen investigations was subsequently performed. This analysis was performed on 42 congenital and developmental cataract patients listed consecutively on the paediatric ophthalmic clinic database; 10 of these had undergone TORCH screen testing. All TORCH screen investigations were performed between 2008 and 2015. Outcomes of the TORCH screen investigations received by each patient 
Table 2 Investigation details of patients with congenital or developmental cataract

\begin{tabular}{|c|c|c|c|c|}
\hline Case & Specialities involved & Grade of investigation & Diagnosis & Months without diagnosis \\
\hline 1 & $\mathrm{O}, \mathrm{P}$ & Standard work-up & No & 28 \\
\hline 2 & $\mathrm{O}, \mathrm{P}$ & Standard work-up & No & 34 \\
\hline 3 & $\mathrm{O}, \mathrm{P}$ & Standard work-up & No & 121 \\
\hline 4 & $\mathrm{O}, \mathrm{G}, \mathrm{C}$ & Standard work-up & No & 18 \\
\hline 5 & $\mathrm{O}, \mathrm{P}$ & None & No & 26 \\
\hline 6 & $\mathrm{O}$ & None & No & 41 \\
\hline 7 & $\mathrm{O}, \mathrm{G}$ & None & No & 179 \\
\hline 8 & $\mathrm{O}$ & None & No & 110 \\
\hline 9 & $\mathrm{O}$ & None & No & 101 \\
\hline 10 & $\mathrm{O}, \mathrm{G}, \mathrm{N}$ & Standard work-up & No & 99 \\
\hline 11 & $\mathrm{O}$ & None & No & 97 \\
\hline 12 & $\mathrm{O}, \mathrm{P}, \mathrm{G}$ & Standard work-up & No & 32 \\
\hline 13 & $\mathrm{O}, \mathrm{G}$ & Standard work-up & No & 17 \\
\hline 14 & $\mathrm{O}$ & None & No & 23 \\
\hline 15 & $\mathrm{O}, \mathrm{P}, \mathrm{G}$ & Standard work-up & No & 14 \\
\hline 16 & $\mathrm{O}, \mathrm{P}$ & Standard work-up & No & 38 \\
\hline 17 & $\mathrm{O}, \mathrm{P}, \mathrm{G}, \mathrm{C}, \mathrm{N}, \mathrm{E}$ & Extensive (microarray) & 17q21.31 deletion syndrome & - \\
\hline 18 & $\mathrm{O}, \mathrm{P}, \mathrm{G}$ & Standard work-up & No & 25 \\
\hline 19 & $\mathrm{O}, \mathrm{P}$ & None & No & 47 \\
\hline 20 & $\mathrm{O}, \mathrm{P}$ & Standard work-up & No & 48 \\
\hline 21 & $\mathrm{O}, \mathrm{P}, \mathrm{G}$ & Standard work-up & No & 35 \\
\hline 22 & $\mathrm{O}, \mathrm{P}, \mathrm{G}, \mathrm{C}, \mathrm{EN}$ & Extensive (microarray) & No & 46 \\
\hline 23 & $\mathrm{O}, \mathrm{G}, \mathrm{U}$ & Extensive (microarray) & No & 42 \\
\hline 24 & $\mathrm{O}, \mathrm{P}, \mathrm{G}, \mathrm{C}, \mathrm{N}, \mathrm{E}, \mathrm{PS}, \mathrm{D}$ & Extensive (microarray) & No & 92 \\
\hline 25 & $\mathrm{O}, \mathrm{P}$ & None & No & 49 \\
\hline 26 & $\mathrm{O}, \mathrm{P}, \mathrm{G}, \mathrm{N}, \mathrm{OR}$ & Extensive (microarray, FISH 22Q13) & No & 47 \\
\hline 27 & $\mathrm{O}, \mathrm{P}, \mathrm{G}, \mathrm{N}, \mathrm{OR}, \mathrm{M}, \mathrm{A}, \mathrm{GE}$ & $\begin{array}{l}\text { Extensive (microarray, } \\
\text { TRMU sequencing, cholesterol studies) }\end{array}$ & No & 44 \\
\hline
\end{tabular}

Abbreviations: C, cardiology; D, dentistry; E, endocrinology; G, genetics; GE, gastroenterology; N, neurology; O, ophthalmology; OR, orthopaedics; P, paediatrics (Medicine and Surgery); PS, plastic surgery. Paediatric work-up: basic blood tests (eg, FBC, TFT, LFT, Ca profile etc), TORCH screen (plasma Ig, culture and PCR investigations), karyotyping, urine analysis for reducing substances and organic amino acids. Extensive: investigations (relevant to cataract only) in addition to above - microarray, cholesterol studies, FISH and single-gene sequencing. Additional investigations in brackets.

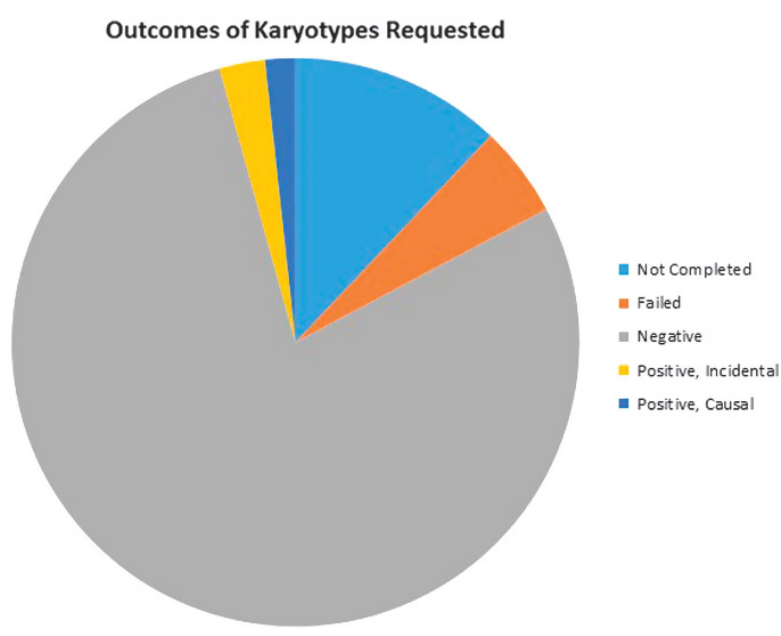

Figure 1 Outcomes of karyotypes requested for the investigation of congenital and developmental cataract patients between 2000 and 2010. The 'not completed' category includes those listed as 'not performed' and 'samples retained for storage only'.

were documented. A TORCH infection was defined as one or more of the following infections: rubella, toxoplasmosis, cytomegalovirus (CMV), herpes simplex virus one and two (HSV1 and HSV2), syphilis and parvovirus. Treponema pallidum allugination assay and rapid plasma reagin investigations were not considered to be part of the TORCH screen, but their results were documented where available.

\section{Next-generation sequencing}

Enrichment of 115 genes associated with congenital and developmental cataract was performed using Agilent SureSelectXT chemistry (Agilent Technologies, Santa Clara, CA, USA) and NGS was performed on the HiSeq 2000 (Illumina Inc, San Diego, CA, USA) platform, as detailed in Gillespie et al. ${ }^{4}$

\section{Results}

What is the clinical utility of standard investigations in identifying the underlying cause of bilateral congenital cataract?

To assess the overall success in diagnosing the underlying cause of bilateral CC -that is, of current standard methods of care - a review of consecutive patients was undertaken between November 2010 and 2012. This identified 
27 patients, of whom 16 were male (59\%), with an age range at presentation between 3 days and 11.9 years. The average age at time of first presentation to the MREH was 38 months (3.2 years). Nine patients (32\%) had a known family history of CC and five (18\%) had a history of consanguinity. The majority of patients $(22 / 27,81 \%)$ received care from other clinical specialities in addition to ophthalmology, most frequently paediatrics $(n=17)$ and genetics $(n=14)$.

Ophthalmic examination revealed that cataract morphology demonstrated considerable diversity. Seven patients exhibited mixed morphology types. Of the 27 patients, 11 (41\%) had other systemic features (Table 1). Learning disability was the most frequent coexisting feature. Patients with systemic features had tended to be investigated more extensively than other patients. Six of the 11 patients with systemic features (patients 17, 22, 23, 24,26 and 27) had undergone extensive investigations $(55 \%)$ and four $(36 \%)$ standard work-up. Nine patients had $(33 \%)$ not undergone any investigations to determine the cause of their cataracts. Of these, 56\% (5/9) had a family history of congenital or developmental cataract.

Only one (patient 17) received a precise diagnosis for their cataract from routine examination and investigation, a diagnosis of 17q21.31 microdeletion syndrome made through microarray analysis. The remaining 26 patients remained awaiting diagnosis for an average of 56 months. All six patients receiving extensive investigations underwent aCGH, which yielded only one diagnosis (patient 17). Chromosome 17q21.31 microdeletion syndrome (also known as Koolen De Vries syndrome) is a multisystem genomic disorder caused by a recurrent 600-kb-long deletion, or haploinsufficiency of the chromatin modifier gene KANSL1, which maps to that region. KANSL1 gene haploinsufficiency is necessary and sufficient to cause the full spectrum of the 17q21.31 microdeletion syndrome. However, bilateral cataracts have not been described so far as part of the clinical spectrum caused by the microdeletion or KANSL1 mutations, ${ }^{5}$ therefore it is uncertain whether the microdeletion found in our patient is the cause of the bilateral cataracts or an additional finding. Descriptions of further patients with Koolen De Vries syndrome and bilateral cataracts would be necessary to confirm this hypothesis.

Fifteen of the study cohort subsequently underwent testing of a panel of cataract genes using NGS. A definitive diagnosis was identified in $60 \%(9 / 15)$ of these patients (Table 3 ), including patient 10 who was found to have a homozygous mutation in the CYP27A1 gene, which is associated with Cerebrotendinous Xanthomatosis.

\section{Clinical utility of chromosome analysis}

Karyotyping was undertaken on the majority of children referred to genetics with bilateral congenital cataracts up until 2014, although some had also undergone array CGH analysis. A review of the results of the cytogenetic analyses undertaken between 2000 and 2010, over which period 116 karyotype investigations were requested for patients with CC (including 18 of the patients in this study). Of these 96 (83\%) were processed, the remaining karyotypes were listed as 'not performed' $(n=9,8 \%)$, 'failed' $(n=6,5 \%)$ or 'storage only' ( $n=5,4 \%$; Figure 1$)$. Ninety-six per cent of processed karyotypes $(n=91)$ returned negative (normal) results. Only $4 \%(n=5)$ of karyotypes yielded abnormal results.

Table 3 Results of NGS testing after its introduction on 01/04/2014 until 01/05/2015

\begin{tabular}{|c|c|c|c|c|c|}
\hline Case & $\begin{array}{l}\text { Mutation } \\
\text { detected? }\end{array}$ & Gene & $\begin{array}{l}\text { Syndromic / } \\
\text { non-syndromic }\end{array}$ & Mutation & Protein change \\
\hline $1^{4}$ & Yes & GALK1 & Syndromic & c.727 T > C (Homozygous) & p.(Cys243Arg) \\
\hline 4 & Yes & GJA3 & Non-syndromic & c.578 T > C (Heterozygous) & p.(Phe193Ser) \\
\hline 7 & Yes & CRYAA & Non-syndromic & c.34C > T (Heterozygous) & p.(Arg12Cys) \\
\hline 10 & Yes & CYP27A1 & Syndromic & c. $1184+1 G>A$ (Homozygous) & - \\
\hline 12 & Yes & $B C O R$ & Syndromic & c.4639C > T (Heterozygous) & p.(Arg1547Ter) \\
\hline 13 & No & - & - & - & - \\
\hline 14 & No & - & - & - & - \\
\hline $16^{4}$ & Yes & GJA8 & Non-syndromic & c.1273C > T (Homozygous) & p.(Arg425X) \\
\hline $17^{4}$ & No & - & - & - & - \\
\hline $20^{4}$ & Yes & $S C 5 D$ & Syndromic & $\begin{array}{l}\text { c. } 479 \mathrm{C}>\mathrm{G}, \mathrm{c} 630 \mathrm{C}>\mathrm{A} \text { (Compound } \\
\text { Heterozygous) }\end{array}$ & $\begin{array}{l}\text { p.(Lys282Glu), } \\
\text { p.ASP210Glu }\end{array}$ \\
\hline $21^{4}$ & Yes & ЕРHA2 & Non-syndromic & c. 855 A > G (Heterozygous) & p.(Lys282Glu) \\
\hline 22 & No & - & - & - & - \\
\hline 23 & Yes & GALT & Syndromic & c.997C > T (Heterozygous) & p.(Arg333Trp) \\
\hline 26 & No & - & - & - & - \\
\hline $27^{4}$ & Yes & CYP51A1 & Syndromic & c.1263G > A, c.935 T > C (Compound Heterozygous) & p.(Typ421X), p.Ile312Thr \\
\hline
\end{tabular}


Likely incidental findings: (i) 45X (Turner syndrome) (ii) $46, X Y, \operatorname{inv}(7)(p 15 p 22)$ and (iii) $46, X X, t(9 ; 20)$ (p13.1;p13)mat,

Expected causative karyotype abnormalities: (iv) Trisomy 21 (Down syndrome);

Unexpected, potentially causative abnormalities (v) 46,XX,inv(13)(q12.3q22.3),add(15)(p13).ish der(15) $\mathrm{t}(6 ; 15)(\mathrm{q} 25 ; \mathrm{p} 13)(6 \mathrm{qter}+, \mathrm{wcp} 6+)$.

Thus, of the five positive results, only two were considered potentially causative of cataract. In a study by Stephen et al, $7.8 \%$ of children with Down syndrome had congenital cataract. ${ }^{6}$ This produces an overall diagnostic rate of $2 \%(2 / 96)$. The remaining three positive findings were judged to be non-causal.

\section{TORCH screening}

Since TORCH screen investigation did not yield any diagnoses in the 27 patients in the study cohort a wider retrospective analysis of TORCH screen results was undertaken to further explore clinical utility (Table 4). Forty-two patients listed consecutively on the paediatric ophthalmic clinic database with bilateral CC were selected for case review (including 18 of the patients in this study). They had presented over a 7 year time frame between 2008 and 2015. Twenty-four percent $(n=10)$ of these were identified as undergoing previous investigation for congenital or antenatal TORCH infection. The average age of patients undergoing TORCH screening was 3.9 years as of April 2014, which is on average younger than those not investigated (3.9 years vs 8.6 years respectively, ages as of April 2014). This indicates TORCH screen was preferentially performed on patients developing cataracts at a younger age. Of the 10 patients undergoing TORCH screening, $20 \%(n=2)$ had a known family history of congenital or developmental cataract and $30 \%(n=3)$ parental consanguinity. The majority of cases $(n=7,70 \%)$ had systemic features in addition to cataract.

The immunoglobulin titres of all patients were measured, with the exception of patient 8 (Table 4). Syphilis immunoglobulin titres were measured in only three patients, unlike the remaining organisms (toxoplasma, rubella virus, CMV, parvovirus, and HSV1 and HSV2) that were routinely investigated in the majority of patients. In contrast to immunoglobulin titres, CMV and HSV PCR investigations were conducted in only three cases. Of the 10 patients undergoing a TORCH screen investigation, $7(70 \%)$ had negative results. Positive results were reported in patients 2 (positive HSV IgG), 5 (positive parvovirus IgG, toxoplasma IgG and CMV IgG) and 6 (positive toxoplasma IgG). Placental transport of $\operatorname{IgG}$ is likely to have accounted for these positive findings given IgM

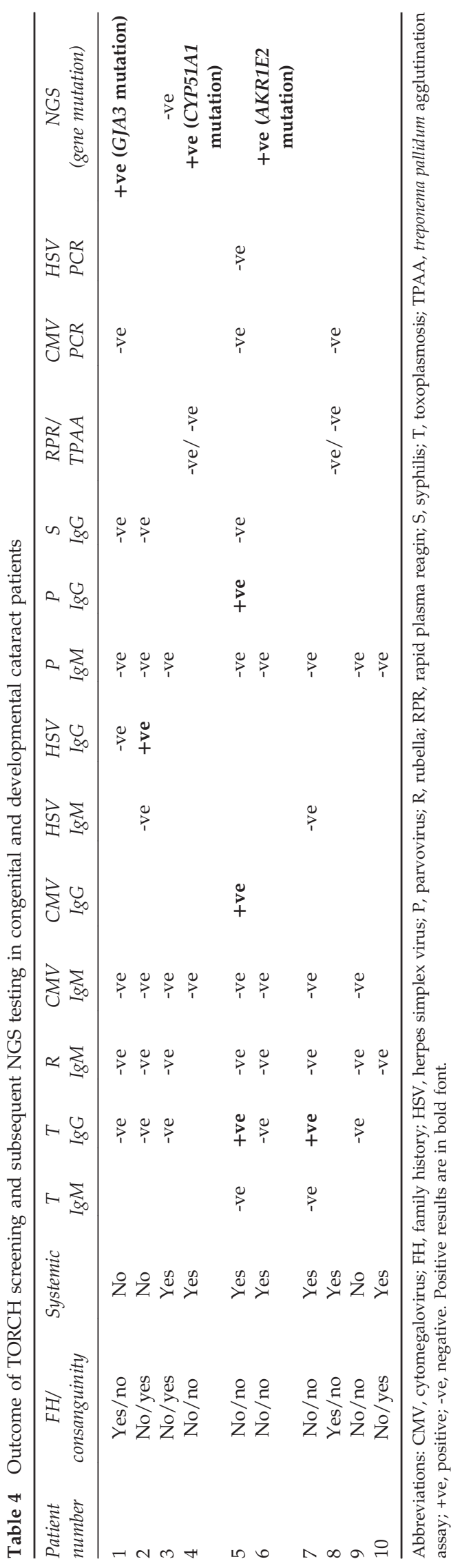


titres and PCR testing all returned negative results in these same patients.

\section{Discussion}

Paediatric cataract has a wide range of aetiologies. Underlying environmental causes include antenatal infection, trauma and chemical or drug exposure. Over $50 \%$ of cases are believed to have a genetic basis and mutations affecting over 110 genes have been described. ${ }^{4}$ Accurate diagnosis is important to provide information to parents, to avoid unnecessary investigations, to facilitate accurate genetic counselling and, potentially, to guide treatment and health surveillance. In the future, genotype-phenotype correlation may also allow for more accurate prognoses. 4

Current standard screening for patients with bilateral congenital and developmental cataracts includes a range of diagnostic tests, although a precise standard diagnostic pathway has not been well-delineated. ${ }^{2}$ This case series demonstrates that current investigative strategies yield a very poor diagnostic return in the majority of patients. Just one out of 27 patients (4\%) studied between 2010 and 2012 had the cause of their cataracts potentially determined using current investigative techniques. In addition to cataracts, this female patient, aged 15 at the time of this study, had global developmental delay and recurrent epileptic seizures. Her diagnosis of 17q21.31 microdeletion syndrome was made by aCGH and was confirmed before the appearance of developmental cataracts. In the majority of patients involved in this study, the cataracts remained of unknown aetiology, despite 10 of the remaining 26 (38\%) undiagnosed patients having systemic problems in addition to their cataracts.

It is of note that testing for galactosaemia in our cohort involved only urinalysis for reducing substances. In fact, this will detect only classical galactosaemia due to GALT deficiency (OMIM*60699) and will not reliably detect galactokinase deficiency (OMIM*230200). The latter is more likely to present as isolated congenital cataracts, and will be missed if screening of plasma galactokinase is not carried out, whereas infants with classical galactosaemia usually present with liver failure in the first week of life, therefore prompting the diagnosis. Currently, screening for galactosaemia is not included in the UK neonatal screening programme. Analysis of the GALT and GALK genes is, however, available as single-gene tests and may be carried out if biochemical testing indicates a galactose disorder.

A low-diagnostic rate for patients with $\mathrm{CC}$ has been demonstrated in other, larger case series, including those conducted by Rahi et $a l^{7}$ and Haargaard et al. ${ }^{8}$ Rahi et $a l^{7}$ reported that no cause of cataract could be identified in
$92 \%$ of unilateral and $38 \%$ of bilateral cataract. Similarly, Haargaard et $a l^{8}$ did not find a cause in $50 \%$ of bilateral and $87 \%$ of unilateral congenital or infantile cataracts.

This study therefore shows that over a decade after these reports, current diagnostic rates for bilateral CC remain disappointing.

This is the first study that quantifies the limited diagnostic capabilities of standard testing before the introduction of NGS to clinical practice in April 2014. The average time awaiting diagnosis was 56 months with only one patient actually receiving a diagnosis, despite $18 / 27(67 \%)$ receiving the standard work-up or extensive investigations.

Our results also demonstrate the variability of care pathways for patients with CC. The majority $(n=22,81 \%)$ received care from a variety of specialities in addition to ophthalmology, underlining both the heterogeneity of CC and the burden of care for these patients. There was variability and inconsistency in the patterns of investigations exemplified by the many patients with a positive family history (patient numbers $3,4,5,7,8,11$, 13, 21 and 25) indicating a clear genetic diagnosis but who nevertheless underwent the standard battery of tests with negative results. This included four (patients 3, 4, 13 and 21) who underwent the full standard work-up plus additional extensive investigations (Table 2) with negative results. Nine of 27 patients did not undergo any investigations, despite this being the current accepted standard.

In the current study, we confirm that, when an integrated paediatric genetic team implements NGS in the diagnostic pathway, this can deliver positive diagnosis in a majority of patients with CC. While six patients in this study underwent NGS panel testing as part of a research study, ${ }^{4}$ a further nine patients underwent NGS panel testing following its introduction as a diagnostic service. Overall, therefore 15/27 patients had undergone NGS cataract panel testing and in this cohort, a diagnosis was found in 60\% (9/15) of these patients (Table 3). As the cases were seen consecutively, this should have eliminated any bias which may have been introduced by selecting particular patients for study with NGS. Diagnoses with systemic associations included oculocardiofacial syndrome (patient 12), galactosaemia (patient 23) and cerebrotendinous xanthomatosis (CTX, patient 10), a potentially treatable metabolic condition. ${ }^{9}$ Mutations associated with isolated autosomal dominant congenital cataract were found in several patients (patients 4, 7 and 16), so in these patients systemic involvement could be excluded and appropriate genetic counselling given to the families.

Overall, this study demonstrates that current standard investigation for patients with bilateral CC is of low clinical utility in the majority of cases as a means of 
identifying a diagnosis. We perhaps need to challenge the views that investigations such as a TORCH screen needs to be undertaken in every patient when there are no other clinical indications. Congenital TORCH infection normally shows other features such as microcephaly, thrombocytopenia or hepatomegaly, with cataracts an uncommon manifestation. ${ }^{10,11}$ Infants with classical galactosaemia present with liver derangement and though exclusion of galactokinase deficiency remains important, infants with this condition have a milder disorder. This condition could be excluded as part of a gene panel. Different guidelines for standard care and low return of positive results lead to inconsistent investigative patterns and, as demonstrated by Gillespie et al can lead to a delay in the treatment and management. ${ }^{9}$ The work suggests that NGS cataract panel testing has the power to offer considerably greater diagnostic efficacy (although currently it must be noted that this technique will not always reliably detect gene deletions and is not a substitute for aCGH in cases where there are cataracts plus additional malformations, growth or developmental problems). One other consideration is whether there are any negative impacts on parents of receiving an early genetic diagnosis through NGS, especially if this reveals an unexpected syndromic cause. What is now required, however, is a realistic assessment of the relative value-formoney of NGS technology within an affordable and deliverable care model for bilateral CC that can be judged against the steadily increasing financial pressure on clinical services to ensure both optimal allocation of resources and cost-effectiveness and also fulfil patients' needs.

\section{Summary}

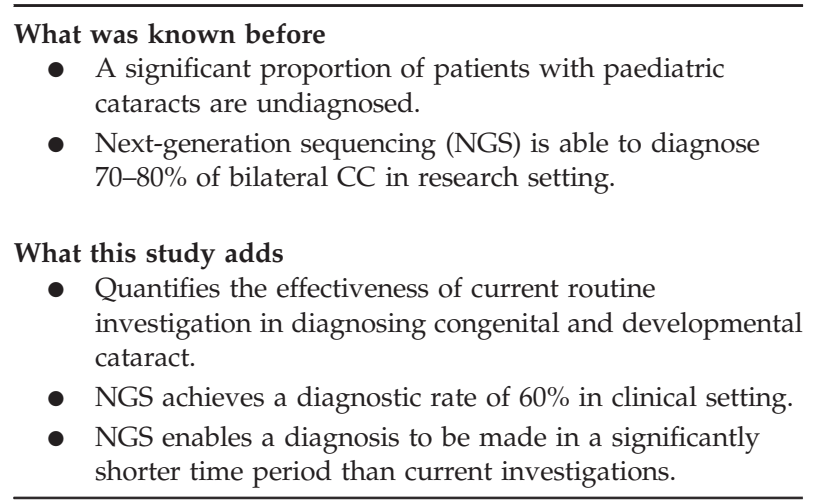

\section{Conflict of interest}

The authors declare no conflict of interest.

\section{Acknowledgements}

This work was funded by Fight for Sight, grant number: 1831. We acknowledge the support of the Manchester Academic Health Science Centre, the Manchester Biomedical Research Centre and Haelo. The funding organization had no role on the design or conduct of this research.

\section{References}

1 Rahi JS, Dezateux C. Measuring and interpreting the incidence of congenital ocular anomalies: lessons from a national study of congenital cataract in the UK. Invest Ophthalmol Vis Sci 2001; 42(7): 1444-1448.

2 Lloyd IC, Goss-Sampson M, Jeffrey BG, Kriss A, Russell-Eggitt I, Taylor D. Neonatal cataract: aetiology, pathogenesis and management. Eye (Lond) 1992; 6(Pt 2): 184-196.

3 Wirth MG, Russell-Eggitt IM, Craig JE, Elder JE, Mackey DA. Aetiology of congenital and paediatric cataract in an Australian population. Br J Ophthalmol 2002; 86(7): 782-786.

4 Gillespie RL, O'Sullivan J, Ashworth J, Bhaskar S, Williams S, Biswas S et al. Personalized diagnosis and management of congenital cataract by next-generation sequencing. Ophthalmology. Ophthalmology 2014; 121 (11): 2124-2137. (e1-2).

5 Moreno-Igoa M, Hernandez-Charro B, Bengoa-Alonso A, Perez-Juana-del-Casal A, Romero-Ibarra C, Nieva-Echebarria B et al. KANSL1 gene disruption associated with the full clinical spectrum of $17 \mathrm{q} 21.31$ microdeletion syndrome. BMC Med Genet 2015; 16: 68.

6 Stephen E, Dickson J, Kindley AD, Scott CC, Charleton PM. Surveillance of vision and ocular disorders in children with Down syndrome. Dev Med Child Neurol 2007; 49(7): 513-515.

7 Rahi JS, Dezateux C. Group tBCCI. Congenital and infantile cataract in the United Kingdom: underlying or associated factors. Invest Ophthalmol Vis Sci 2000; 41 (8): 2108-2114.

8 Haargaard B, Wohlfahrt J, Fledelius HC, Rosenberg T, Melbye M. A nationwide Danish study of 1027 cases of congenital/infantile cataracts: etiological and clinical classifications. Ophthalmology 2004; 111(12): 2292-2298.

9 Gillespie RL, Urquhart J, Anderson B, Williams S, Waller S, Ashworth $\mathrm{J}$ et al. Next-generation sequencing in the diagnosis of metabolic disease marked by pediatric cataract. Ophthalmology 2016; 123(1): 217-220.

10 Coats DK, Demmler GJ, Paysse EA, Du LT, Libby C. Ophthalmologic findings in children with congenital cytomegalovirus infection. J AAPOS 2000; 4(2): 110-116.

11 Arun V, Noble AG, Latkany P, Troia RN, Jalbrzikowski J, Kasza $\mathrm{K}$ et al. Cataracts in congenital toxoplasmosis. J AAPOS 2007; 11(6): 551-554. 